\title{
Universal abelian covers of rational surface singularities and multi-index filtrations *
}

\author{
A. Campillo $\quad$ F. Delgado ${ }^{\dagger} \quad$ S.M. Gusein-Zade ${ }^{\ddagger}$
}

In [1] and [2], there were computed the Poincaré series of some (multi-index) filtrations on the ring of germs of functions on a rational surface singularity. These Poincaré series were written as the integer parts of certain fractional power series, an interpretation of whom was not given. Here we show that, up to a simple change of variables, these fractional power series are specializations of the equivariant Poincaré series for filtrations on the ring $\varnothing_{\widetilde{\mathcal{S}}, 0}$ of germs of functions on the universal abelian cover $(\widetilde{\mathcal{S}}, 0)$ of the surface $(\mathcal{S}, 0)$. We compute these equivariant Poincaré series. From another point of view universal abelian covers of rational surface singularities were studied in [6].

Let $(\mathcal{S}, 0)$ be an isolated complex rational surface singularity and let $\pi$ : $(X, \mathcal{D}) \rightarrow(\mathcal{S}, 0)$ be a resolution of it (not necessarily the minimal one). Here $X$ is a smooth complex surface, the exceptional divisor $\mathcal{D}=\pi^{-1}(0)$ is a normal crossing divisor on $X$, all components $E_{\sigma}(\sigma \in \Gamma)$ of the exceptional divisor $\mathcal{D}$ are isomorphic to the complex projective line $\mathbb{C P}^{1}$ and the dual graph of the resolution is a tree.

Let $\varnothing_{\mathcal{S}, 0}$ be the ring of germs of analytic functions on $(\mathcal{S}, 0)$. For $\sigma \in \Gamma$, i.e. for a component $E_{\sigma}$ of the exceptional divisor, and for $f \in \varnothing_{\mathcal{S}, 0}$, let $v_{\sigma}(f)$ be the order of zero of the lifting $f \circ \pi$ of the function $f$ to the space $X$ of the resolution along the component $E_{\sigma}$. Let us choose several components $E_{1}, \ldots, E_{s}$ of the exceptional divisor $\mathcal{D}(\{1, \ldots, s\} \subset \Gamma)$. The valuations $v_{1}, \ldots, v_{s}$ define a multi-index filtration $\{J(\underline{v})\}$ on the ring $\varnothing_{\mathcal{S}, 0}$ : for $\underline{v}=\left(v_{1}, \ldots, v_{s}\right) \in \mathbb{Z}_{\geq 0}^{s}$, $J(\underline{v})=\left\{f \in \varnothing_{\mathcal{S}, 0}: \underline{v}(f) \geq \underline{v}\right\}$ (here $\underline{v}(f)=\left(v_{1}(f), \ldots, v_{s}(f)\right) \in \mathbb{Z}_{\geq 0}^{s}, \underline{v}^{\prime} \geq \underline{v}$

*Math. Subject Class. 14H20, 14J17, 32S25. Keywords: universal abelian covers, rational surface singularities, Poincaré series.

${ }^{\dagger}$ First two authors were partially supported by the grant MTM2004-00958. Address: University of Valladolid, Dept. of Algebra, Geometry and Topology, 47011 Valladolid, Spain. E-mail: campillo@agt.uva.es, fdelgado@agt.uva.es

${ }^{\ddagger}$ Partially supported by the grants RFBR-007-00593, INTAS-05-7805, NWO-RFBR 047.011.2004.026, and RFBR-JSPS 06-01-91063. Address: Moscow State University, Faculty of Mathematics and Mechanics, Moscow, GSP-2, 119992, Russia. E-mail: sabir@mccme.ru 
if and only if $v_{i}^{\prime} \geq v_{i}$ for all $\left.i=1, \ldots, s\right)$. In [1], there was computed the Poincaré series $P\left(t_{1}, \ldots, t_{s}\right)$ of this filtration (the definition of the Poincaré series of a multi-index filtration can be found e.g. in [1, 2, 3]). Let $\left(E_{\sigma} \circ E_{\delta}\right)$ be the intersection matrix of the components of the exceptional divisor. For $\sigma \neq \delta$, the intersection number $E_{\sigma} \circ E_{\delta}$ is equal to 1 if the components $E_{\sigma}$ and $E_{\delta}$ intersect (at one point) and is equal to zero if they don't intersect; the self-intersection number $E_{\sigma} \circ E_{\sigma}$ of each component $E_{\sigma}$ is a negative integer. Let $d=\operatorname{det}\left(-\left(E_{\sigma} \circ E_{\delta}\right)\right)$ and let $\left(m_{\sigma \delta}\right)=-\left(E_{\sigma} \circ E_{\delta}\right)^{-1}$. All entries $m_{\sigma \delta}$ are positive and $m_{\sigma \delta} \in(1 / d) \mathbb{Z}$. For $\sigma \in \Gamma$, let $\underline{m}_{\sigma}:=\left(m_{\sigma 1}, \ldots, m_{\sigma s}\right) \in \mathbb{Q}_{\geq 0}^{s}$.

Let $\dot{E}_{\sigma}$ be the "smooth part" of the component $E_{\sigma}$ in the exceptional divisor $\mathcal{D}$, i.e., $E_{\sigma}$ minus intersection points with all other components of the exceptional divisor $\mathcal{D}$.

For a fractional power series $S\left(t_{1}, \ldots, t_{s}\right) \in \mathbb{Z}\left[\left[t_{1}^{1 / d}, \ldots, t_{s}^{1 / d}\right]\right]$, let Int $S\left(t_{1}, \ldots, t_{s}\right)$ be its "integer part", i.e., the sum of all monomials from $S\left(t_{1}, \ldots, t_{s}\right)$ with integer exponents. In [1] it was shown that

$$
P\left(t_{1}, \ldots, t_{s}\right)=\operatorname{lnt} \prod_{\sigma \in \Gamma}\left(1-\underline{t}^{\underline{\underline{m}}}\right)^{-\chi\left(\dot{E}_{\sigma}\right)},
$$

where $\underline{t}^{\underline{m}}:=t_{1}^{m_{1}} \cdot \ldots \cdot t_{s}^{m_{s}}, \chi(X)$ is the Euler characteristic of the space $X$.

A similar formula was obtained in [2] for the Poincaré series of the multiindex filtration on the ring $\varnothing_{\mathcal{S}, 0}$ defined by orders of a function germ on irreducible components of a curve $(C, 0) \subset(\mathcal{S}, 0)$.

In [1], the fractional power series

$$
Q(\underline{t})=\prod_{\sigma \in \Gamma}\left(1-\underline{t}^{\underline{m}} \sigma\right)^{-\chi\left(\dot{E}_{\sigma}\right)}
$$

(and a similar one in 22]) participated as a formal expression convenient to write the formula (11) for the Poincaré series $P\left(t_{1}, \ldots, t_{s}\right)$. There was no interpretation of it.

In [3], there was defined an equivariant Poincaré series for an "equivariant" filtration on the ring $\varnothing_{V, 0}$ of germs of functions on a germ $(V, 0)$ of a complex analytic variety with an action of a finite group $G$. This Poincaré series was computed for a divisorial filtration on the ring $\varnothing_{\mathbb{C}^{2}, 0}$ and for the filtration defined by branches of a $G$-invariant plane curve singularity $(C, 0) \subset\left(\mathbb{C}^{2}, 0\right)$ were the plane $\mathbb{C}^{2}$ was equipped with a $G$-action.

Let $p:(\widetilde{\mathcal{S}}, 0) \rightarrow(\mathcal{S}, 0)$ be the universal abelian cover of the surface singularity $(\mathcal{S}, 0)$ : see e.g. [5, 6]. One can describe it in the following way. Let $\mathrm{G}=H_{1}(\mathcal{S} \backslash\{0\})$ be the first homology group of the (nonsingular) surface $\mathcal{S} \backslash\{0\}$. The order of the group $G$ is equal to the determinant $d$ of the minus 
intersection matrix $-\left(E_{\sigma} \circ E_{\delta}\right)$ and moreover $G$ is the cokernel $\mathbb{Z}^{\Gamma} / \operatorname{Im} I$ of the $\operatorname{map} I: \mathbb{Z}^{\Gamma} \rightarrow \mathbb{Z}^{\Gamma}$ defined by this matrix.

The group $G$ acts on the germ $(\widetilde{\mathcal{S}}, 0)$ and the restriction $\left.p\right|_{\widetilde{\mathcal{S}} \backslash\{0\}}$ of the map $p$ to the complement of the origin is a (usual, nonramified) covering $\widetilde{\mathcal{S}} \backslash\{0\} \rightarrow \mathcal{S} \backslash\{0\}$ with the structure group $G$. One can lift the map $p$ to a (ramified) covering $p:(\widetilde{X}, \widetilde{\mathcal{D}}) \rightarrow(X, \mathcal{D})$ where $\widetilde{X}$ is a normal surface (generally speaking not smooth) and $\widetilde{X} \backslash \widetilde{\mathcal{D}} \cong \widetilde{\mathcal{S}} \backslash\{0\}$ :

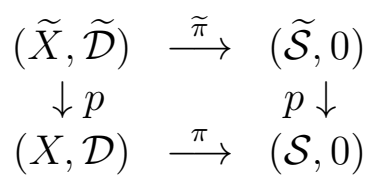

(one can define $\widetilde{X}$ as the normalization of the fibre product $X \times{ }_{\mathcal{S}} \widetilde{\mathcal{S}}$ of the varieties $X$ and $\widetilde{\mathcal{S}}$ over $\mathcal{S}$ ).

Let $g_{\sigma}, \sigma \in \Gamma$ be the element of the group $G$ represented by the loop in $X \backslash \mathcal{D}$ going around the component $E_{\sigma}$ in the positive direction. The group $G$ is generated by the elements $g_{\sigma}$ for all $\sigma \in \Gamma$. For a point $x \in \dot{E}_{\sigma}$ and for a point $\widetilde{x}$ from the preimage $p^{-1}(x)$ of it, locally, in a neighbourhood of the point $\widetilde{x}$, the map $p: \widetilde{D} \rightarrow \mathcal{D}$ is an isomorphism and the map $p: \widetilde{X} \rightarrow X$ is a ramified (over $\mathcal{D}$ ) covering, the order $d_{\sigma}$ of which coincides with the order of the generator $g_{\sigma}$ of the group $G$.

Lemma 1 The order $d_{\sigma}$ of the element $g_{\sigma} \in G$ is the minimal natural $k$ such that $k m_{\delta \sigma}$ is an integer for all $\delta \in \Gamma$.

Proof. This follows immediately from the fact that $\mathbb{Z}^{\Gamma} / \operatorname{Im} I \cong \operatorname{Im} m / \mathbb{Z}^{\Gamma}$ where $m: \mathbb{Z}^{\Gamma} \rightarrow \mathbb{Q}^{\Gamma}$ is the map given by the matrix $\left(m_{\sigma \delta}\right)$ (i.e. minus the inverse of the map $I$ ).

Let $R(G)$ be the ring of (virtual) representations of the group $G$. For $\sigma \in \Gamma$, let $\alpha_{\sigma}$ be the one-dimensional representation $G \rightarrow \mathbb{C}^{*}=\mathbf{G L}(1, \mathbb{C})$ of the group $G$ defined by $\alpha_{\sigma}\left(g_{\delta}\right)=\exp \left(-2 \pi \sqrt{-1} m_{\sigma \delta}\right)$ (here the minus sign reflects the fact that the action of an element $g \in G$ on the ring $\varnothing_{\widetilde{\mathcal{S}}, 0}$ is defined by $\left.(g \cdot f)(x)=f\left(g^{-1}(x)\right)\right)$.

Let us choose any component $\widetilde{E}_{i}$ of the preimage $p^{-1}\left(E_{i}\right)$ of the component $E_{i}$ and let $\widetilde{v}_{i}$ be the corresponding divisorial valuation on the ring $\varnothing_{\widetilde{\mathcal{S}}, 0}$. On the space $\bigcup_{\alpha} \varnothing_{\mathcal{S}, 0}^{\alpha}$ of all $G$-equivariant functions on $(\widetilde{\mathcal{S}}, 0)$ ( $\alpha$ runs over all nonequivalent 1-dimensional representations of the group $G$ ) the valuation $\widetilde{v}_{i}$ does not depend on the choice of the component $\widetilde{E}_{i}$.

In [3], there was defined the equivariant Poincaré series of the multi-index filtration defined by the divisorial valuations $\widetilde{v}_{1}, \ldots, \widetilde{v}_{s}$. 
Theorem 1 The equivariant Poincaré series $P^{G}\left(t_{1}, \ldots, t_{s}\right)$ of the s-index filtration defined by the set of divisorial valuations $\left\{\widetilde{v}_{1}, \ldots, \widetilde{v}_{s}\right\}$ is given by the formula:

$$
\begin{aligned}
P^{G}\left(t_{1}, \ldots, t_{s}\right) & =\prod_{\sigma \in \Gamma}\left(1-\alpha_{\sigma} \underline{t}^{\underline{d} \underline{m}_{\sigma}}\right)^{-\chi\left(\dot{E}_{\sigma}\right)} \\
& =\prod_{\sigma \in \Gamma}\left(1-\alpha_{\sigma} t_{1}^{d_{1} m_{1 \sigma}} \cdot \ldots \cdot t_{s}^{d_{s} m_{s \sigma}}\right)^{-\chi\left(\dot{E}_{\sigma}\right)}
\end{aligned}
$$

For a power series $S\left(t_{1}, \ldots, t_{s}\right)=\sum_{\underline{v} \in \mathbb{Z}_{\geq 0}^{s}} s_{\underline{v}} \underline{t} \underline{v} \in R(G)\left[\left[t_{1}, \ldots, t_{s}\right]\right](R(G)$ is the ring of representations of the group $G)$, let its reduction red $S\left(t_{1}, \ldots, t_{s}\right)$ be the series $\sum_{\underline{v} \in \mathbb{Z}_{\geq 0}^{s}}\left(\operatorname{dim} s_{\underline{v}}\right) \underline{t} \underline{v} \in \mathbb{Z}\left[\left[t_{1}, \ldots, t_{s}\right]\right]$.

Corollary. One has red $P^{G}\left(t_{1}, \ldots, t_{s}\right)=Q\left(t_{1}^{d_{1}}, \ldots, t_{s}^{d_{s}}\right)$, where $Q(\underline{t})$ is the fractional power series defined by (2).

Proof of Theorem 1 For short we shall say that an effective divisor on $\dot{\mathcal{D}}=\bigcup \dot{E}_{\sigma}\left(\right.$ or on $\left.\dot{\widetilde{\mathcal{D}}}=p^{-1}(\dot{\mathcal{D}})\right)$ is Cartier if it is the intersection with $\dot{\mathcal{D}}$ (or with $\dot{\widetilde{\mathcal{D}}})$ of the strict transform of a Cartier divisor on $(\mathcal{S}, 0)$ (or on $(\widetilde{\mathcal{S}}, 0)$ ). From [3] it follows that the equivariant Poincaré series $P^{G}(\underline{t})$ is equal to the integral with respect to the Euler characteristic of the monomial $\alpha \underline{t} \underline{\underline{v}}$ over the space of $G$-invariant effective Cartier divisors on $\dot{\widetilde{\mathcal{D}}}$. Here $\alpha \in R(G)$ and $\underline{\widetilde{v}} \in \mathbb{Z}_{\geq 0}^{s}$ are functions (in fact semigroup homomorphisms) on the space of $G$-invariant Cartier divisors on $\dot{\widetilde{\mathcal{D}}}$ : a $G$-invariant Cartier divisor defines the orders of zero of the corresponding ( $G$-equivariant) function along the components $\dot{E}_{i}$ and also the corresponding 1-dimensional representation of the group $G$.

Thus to compute the equivariant Poincaré series $P^{G}(\underline{t})$ one has to describe the space of $G$-invariant effective Cartier divisors on $\stackrel{\dot{\mathcal{D}}}{\text { and the corresponding }}$ functions $\underline{v}$ and $\alpha$ on it.

Lemma 2 Any G-invariant effective divisor on $\dot{\widetilde{\mathcal{D}}}$ is a Cartier divisor.

Proof. It is sufficient to show this for the divisor $\sum_{\widetilde{x} \in p^{-1}(x)} \widetilde{x}$ for a point $x \in \dot{E}_{\sigma}$, i.e. for the $G$-orbit of a point from $\dot{\widetilde{E}}_{\sigma}$. The isotropy group $G_{\widetilde{x}}$ of a point $\widetilde{x} \in p^{-1}(x)$ is the cyclic subgroup of the group $G$ of order $d_{\sigma}$ generated by the element $g_{\sigma}$ (this element acts trivially on $p^{-1}\left(E_{\sigma}\right)$ ). 
Let us take the germ at the point $x$ of a smooth curve $L_{\sigma}$ on $(X, \mathcal{D})$ transversal to the exceptional divisor $\mathcal{D}$. By the Artin criterion (see, e.g., [7, Lemma on page 156), the divisor $d \cdot L_{\sigma}$ is the strict transform of a Cartier divisor on $(\mathcal{S}, 0)$ (in fact already $d_{\sigma} L_{\sigma}$ is one with this property), i.e. there exists a function $f_{\sigma}: \mathcal{S} \rightarrow \mathbb{C}$ such that the strict transform of the divisor $\left\{f_{\sigma}=0\right\}$ is $d \cdot L_{\sigma}$. Let $\bar{f}_{\sigma}=f_{\sigma} \circ \pi$ be the lifting of the function $f_{\sigma}$ to the space $X$ of the resolution and let $\tilde{f}_{\sigma}=f_{\sigma} \circ \pi \circ p$ be the lifting of the function $f_{\sigma}$ to the space $\widetilde{X}$ of the modification of the universal abelian cover $(\widetilde{\mathcal{S}}, 0)\left(\widetilde{f}_{\sigma}\right.$ is a $G$-invariant function on $\tilde{X})$. Let us describe the divisor $\left\{\widetilde{f}_{\sigma}=0\right\}$. Let $\widetilde{L}_{\sigma, \widetilde{x}} \subset \widetilde{X}$ be the germ at the point $\widetilde{x} \in p^{-1}(x)$ of the preimage under the map $p$ of the curve $L_{\sigma} \subset X$.

The order of zero of the function $\widetilde{f}_{\sigma}$ along $\widetilde{L}_{\sigma, \widetilde{x}}$ is equal to $d$. The order of zero of the function $\bar{f}_{\sigma}$ along the component $\dot{E}_{\delta}$ is equal to $d \cdot m_{\sigma \delta}$. The ramification order of the map $p$ over the component $\dot{E}_{\delta}$ is equal to $d_{\delta}$. Therefore the order of zero of the function $\widetilde{f}_{\sigma}=\bar{f}_{\sigma} \circ p$ along the preimage of the component $\dot{E}_{\delta}$ is equal to $d \cdot d_{\delta} \cdot m_{\sigma \delta}$. This (integer) number is divisible by $d$ (since $d_{\delta} m_{\sigma \delta}$ is an integer: see Lemma (1). Therefore the zero divisor of the function $\widetilde{f}_{\sigma}$ is divisible by $d$, i.e. the order of zero of this function along each component of its zero set is divisible by $d$. This means that a root $\sqrt[d]{\widetilde{f}_{\sigma}}$ of degree $d$ of the function $\widetilde{f}_{\sigma}$ (i.e. a branch of this root) is well defined up to multiplication by a root of degree $d$ of $1 G$-equivariant complex analytic function on $\widetilde{X}$ and thus it is the lifting of a $G$-equivariant function on $(\widetilde{\mathcal{S}}, 0)$ (see e.g. [4, page ?]).

Corollary. Each $G$-invariant divisor on the universal abelian cover $(\widetilde{\mathcal{S}}, 0)$ of the rational surface singularity $(\mathcal{S}, 0)$ is a Cartier one.

Lemma 2 means that the space of $G$-invariant effective Cartier divisors on $\dot{\widetilde{\mathcal{D}}}$ is in one to one correspondence with the space of all effective divisors on $\dot{\widetilde{\mathcal{D}}}$. As it follows from the proof of Lemma 2, the order of zero of the $G$-equivariant function $\widetilde{f}_{\sigma}$ (corresponding to one point $x \in \dot{E}_{\sigma}$ ) along the component $\widetilde{E}_{i}$ is equal to $d_{i} m_{\sigma i}$. One has to find the (one-dimensional) representation $\alpha_{\sigma}$ with respect to which the function $\tilde{f}_{\sigma}$ is $G$-equivariant.

\section{Lemma 3}

$$
\alpha_{\sigma}\left(g_{\delta}\right)=\exp \left(-2 \pi \sqrt{-1} m_{\sigma \delta}\right)
$$

Proof. The element $g_{\delta}$ of the group $G$ acts trivially on the preimage $p^{-1}\left(\dot{E}_{\delta}\right)$ of the component $\dot{E}_{\delta}$ of the exceptional divisor and acts by multiplication by 
$\exp \left(\frac{2 \pi}{d_{\delta}} \sqrt{-1}\right)$ on the normal line to it. The order of zero of the function $\widetilde{f}_{\sigma}$ along the preimage $p^{-1}\left(\dot{E}_{\delta}\right)$ is equal to $m_{\sigma \delta} d_{\delta}$. Therefore

$$
\frac{g_{\delta} \cdot f_{\sigma}}{f_{\sigma}}=\exp \left(-\frac{2 \pi \sqrt{-1} m_{\sigma \delta} d_{\delta}}{d_{\delta}}\right)=\exp \left(-2 \pi \sqrt{-1} m_{\sigma \delta}\right) .
$$

Now Theorem follows from the usual arguments used e.g. in [1, 3]. The space of effective divisors on $\dot{\mathcal{D}}=\bigcup \dot{E}_{\sigma}$ is the direct product of the spaces of effective divisors on the components $\dot{E}_{\sigma}$. Each of the latter ones is the disjoint union of symmetric powers $S^{k} \dot{E}_{\sigma}$ of the component $\dot{E}_{\sigma}$. Therefore

$$
P^{G}\left(t_{1}, \ldots, t_{s}\right)=\prod_{\sigma \in \Gamma}\left(\sum_{k=0}^{\infty} \chi\left(S^{k} \dot{E}_{\sigma}\right) \cdot \alpha_{\sigma}^{k} \underline{t}^{k \underline{d} \underline{m}_{\sigma}}\right)
$$

(this follows from the fact that $\underline{v}$ and $\alpha$ are semigroup homomorphisms). The well-known formula

$$
\sum_{k=0}^{\infty} \chi\left(S^{k} X\right) t^{k}=(1-t)^{-\chi(X)}
$$

implies the equation (3).

A similar result holds for the filtration on the ring $\varnothing_{\widetilde{\mathcal{S}}, 0}$ defined by orders of a function germ on branches of a $G$-invariant curve $(\widetilde{C}, 0) \subset(\widetilde{\mathcal{S}}, 0)$. Let $\widetilde{C}=\bigcup_{i=1}^{r} \widetilde{C}_{i}$ where $\widetilde{C}_{i}$ are irreducible $G$-invariant components of the curve $\widetilde{C}$ (generally speaking each curve $\widetilde{C}_{i}$ consists of several irreducible compoents permuted by the group $G$ ). Each curve $\widetilde{C}_{i}$ is the preimage under the map $p$ of an irreducible curve $C_{i}$ on $(\mathcal{S}, 0)$. The curve $\widetilde{C}=\bigcup_{i=1}^{r} \widetilde{C}_{i}$ defines an $r$-index filtration on the space $\bigcup_{\alpha} \varnothing_{\widetilde{\mathcal{S}}, 0}^{\alpha}$ of $G$-equivariant functions on the surface $(\widetilde{\mathcal{S}}, 0)$ (or on the space $\bigcup_{\alpha} \varnothing_{\widetilde{C}, 0}^{\alpha}$ of $G$-equivariant functions on the cuvre $(\widetilde{C}, 0)$ ). Let $\varphi_{i}:(\mathbb{C}, 0) \rightarrow(\widetilde{\mathcal{S}}, 0)$ be a parametrization (uniformization) of an irreducible component of the curve $\widetilde{C}_{i}$. For a $G$-equivariant function germ $f$, let $\widetilde{w}_{i}(f)$ be the order of zero of the function $f \circ \varphi_{i}$ at the origin: $f \circ \varphi_{i}(\tau)=a \tau^{\widetilde{w}_{i}(f)}+$ terms of higher degree, $a \neq 0$. The valuations $\widetilde{w}_{1}, \ldots, \widetilde{w}_{r}$ define a multi-index filtration in the usual way.

Let $\pi:(X, \mathcal{D}) \rightarrow(\mathcal{S}, 0)$ be a resolution of the surface singularity $(\mathcal{S}, 0)$ which at the same time is an embedded resolution of the curve $(C, 0) \subset(\mathcal{S}, 0)$, 
$C=\bigcup_{i=1}^{r} C_{i}$. Let $\bar{C}_{i}$ be the strict trasnform of the curve $C_{i}$ in $X$. Let $E_{1}, \ldots, E_{s}$ be all the components of the exceptional divisor $\mathcal{D}$ of the resolution. Let $\stackrel{\circ}{E}_{i}$ be the "smooth part" of the component $E_{i}$ in the total transform $\pi^{-1}(C)$ of the curve $C$, i.e. $E_{i}$ minus intersection points with all other components of the total transform $\pi^{-1}(C)$. Let $\underline{m}_{i}=\left(m_{i 1}, \ldots, m_{i s}\right) \in \mathbb{Q}_{\geq 0}^{s}, \underline{d}=\left(d_{1}, \ldots, d_{s}\right) \in \mathbb{Z}_{\geq 0}^{s}$, and a 1-dimensional representation $\alpha$ of the group $G(i=1, \ldots, s)$ be defined as above. The same arguments as in the proof of Theorem 1 imply the following statement.

Theorem 2 The equivariant Poincaré series $P^{G}\left(t_{1}, \ldots, t_{r}\right)$ of the $r$-index filtration defined by the set of valuations $\left\{\widetilde{w}_{1}, \ldots, \widetilde{w}_{r}\right\}$ is given by the formula:

$$
P^{G}\left(t_{1}, \ldots, t_{r}\right)=\left.\left(\prod_{i=1}^{s}\left(1-\alpha_{i} \underline{T}^{\underline{d} \underline{m}_{i}}\right)^{-\chi\left(\stackrel{\circ}{E}_{i}\right)}\right)\right|_{T_{i} \mapsto \prod_{j: \bar{C}_{j} \cap E_{i}=p t} t_{j}}
$$

(here $\underline{T}=\left(T_{1}, \ldots, T_{s}\right)$; in the substitution above, $\prod_{j \in \emptyset} t_{j}$ is supposed to be equal to 1$)$.

\section{References}

[1] Campillo A., Delgado F., Gusein-Zade S.M. Poincaré series of a rational surface singularity. Invent. math. 155, 41-53 (2004).

[2] Campillo A., Delgado F., Gusein-Zade S.M. Poincaré series of curves on rational surface singularities. Comment. Math. Helv., 80, no.1, 95-102 (2005).

[3] Campillo A., Delgado F., Gusein-Zade S.M. On Poincaré series of filtrations on equivariant functions of two variables. Moscow Math. J., 7, no.2, $243-255$ (2007).

[4] Laufer H. B. Normal two-dimensional singularitites. Ann. of Math. Studies, 71. Princeton Univ. Press, Princeton, NJ, 1971.

[5] Neumann W.D., Wahl J. Universal abelian covers of surface singularities. In: Trends in singularities, 181-190, Trends Math., Birkhuser, Basel, 2002 .

[6] Okuma T. Universal abelian covers of rational surface singularities. J. London Math. Soc. (2), 70, no.2, 307-324 (2004). 
[7] Pinkham H. Singularités rationelles de surfaces. In: Séminaire sur les singularités des surfaces. Lecture Notes in Math. 777, 147-178. Springer, Berlin-Heidelberg-New York, 1980. 